\title{
Spinoffs from space
}

\section{Robert Thirsk MDCM SM, Andre Kuipers MD, Chiaki Mukai MD PhD, David Williams MDCM MSc}

Published at www.cmaj.ca on June 15, 2009.

$\infty \quad$ See related review by Williams and colleagues, page 1317

$\mathrm{S}$ ome of the technological advances required for space travel and the understanding gained through space research have proved useful in the practice of medicine on earth. Research into medicine in space, for example, has deepened our understanding of human physiology. Entire journal issues have been devoted to this topic. ${ }^{1}$ Many innovations and techniques for delivering medical care in space have been modified and applied on earth.

\section{Telemedicine}

Providing long-distance care can be difficult at the best of times, but caring for patients in space has added challenges. Spaceflight is the ultimate setting to demonstrate and evaluate telemedicine concepts and procedures. Astronauts with minimal training can obtain diagnostic-quality ultrasonography images and can perform other medical and surgical procedures with remote guidance. ${ }^{2}$ These protocols are now being adapted for remote clinical settings. For example, a procedure for ultrasonography developed for spaceflight is now being used in northern Canada to diagnose pneumothoraces following trauma.

\section{Innovations}

Innovations from space often have applications on earth. ${ }^{4}$ The National Aeronautics and Space Administration (NASA) has documented over 1500 space technologies that have been adapted for societal applications, such as protective clothing for fire fighters, sturdy roofing materials and safer school buses. ${ }^{5}$ Technologies have been transferred for use in transportation, public safety, consumer goods, environmental resources, computer technology and industry. Although Tang, Teflon and Velcro are frequently cited as being spinoffs of the space program, this is untrue. Each of these products existed before spaceflight, but useful applications for each have been found in space.

Health and medicine are perhaps the biggest beneficiaries of these dual-use technologies. Liquid-cooling garments, originally developed to protect astronauts working on the surface of the moon from the scorching heat of a lunar day, are now commonly used in many occupational, medical and athletic settings. ${ }^{6}$ In medicine, these garments are used to treat many conditions, including spinal injuries, multiple sclerosis and sports injuries.

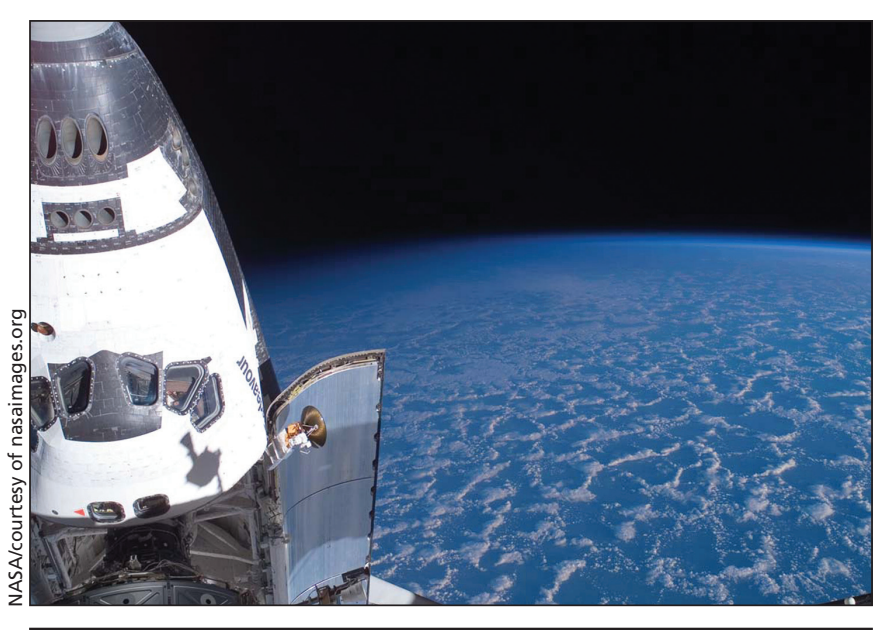

The space shuttle Endeavour is shown against the backdrop of earth's horizon.

A flow cytometer originally developed for use in space is now used to analyze human solid tumours. ${ }^{7}$ The Automated Endoscopic System for Optimal Positioning (AESOP) robot, commonly found in many hospitals, was developed as a spinoff from technologies created at the NASA Jet Propulsion Laboratory. ${ }^{8}$ The turbopump design from the space shuttle's main engines was modified to develop a left ventricular assist device. ${ }^{9}$ Hundreds of similar examples are documented in websites devoted to spinoffs from space technology.

\section{Canadian applications}

Canadian space technology has had important benefits on earth. Imagery from Canada's RADARSAT and other remote sensing satellites has been provided to developing countries to monitor the spread of vector-borne diseases. ${ }^{10}$ The Canadian investment in space robotic technology is benefiting surgical patients. MacDonald, Dettwiler and Associates, the company that built the robot manipulators for the space shuttle and the International Space Station, has partnered with the University of Calgary Medical School to develop a telerobotic microsurgical system called neuroArm. ${ }^{11}$ NeuroArm

From the Canadian Space Agency (Thirsk); the European Space Agency Astronaut Corps (Kuipers); the Japan Aerospace Exploration Agency (Mukai); and the Department of Surgery (Williams), McMaster University, Hamilton, Ont. 
incorporates space control systems and vision technology that allows surgeons to perform intricate microscopic neurosurgical procedures. MacDonald, Dettwiler and Associates is currently developing a third-generation image-guided surgical robot in partnership with the McMaster University Centre for Medical Robotics to enhance the capability of surgeons to perform precise surgical procedures.

This article has been peer reviewed.

Competing interests: David Williams was employed by the Canadian Space Agency from 1992 to 2008. During this time, he performed research into the space environment. None declared for Robert Thirsk, Andre Kuipers and Chiaki Mukai.

Contributors: All of the authors were involved in the drafting and revision of the article and approved the final version submitted for publication.

\section{REFERENCES}

1. Hinghofer-Szalkay H. Traveling space: biological considerations, and the benefits for terrestrial medicine. Curr Pharm Biotechnol 2005;6:251

2. Thirsk R, Williams D, Anvari M. NEEMO 7 Undersea mission. Acta Astronautica 2007;60:512-7.

3. Kirkpatrick AW, Nicolaou S, Rowan K, et al. Thoracic sonography for pneumothorax: the clinical evaluation of an operational space medicine spin-off. Acta Astronaut 2005;56:831-8.

4. Global space sector market trends and drivers 2004. Saint-Hubert (QC): External Relations Directorate, Canadian Space Agency; 2006. Available: www.asccsa.gc.ca/eng/industry/strategic.asp (accessed 2009 May 7).

5. National Aeronautics and Space Administration (NASA). Spinoff Database. Washington (DC): NASA; 2007. Available: www.sti.nasa.gov/spinoff/database (accessed 2009 May 7).

6. Flouris AD, Cheung SS. Design and control optimization of microclimate liquid cooling systems underneath protective clothing. Ann Biomed Eng 2006;34:359-72.

7. Cram LS. Spin-offs from the NASA space program for tumor diagnosis. Cytometry 2001;43:1.

8. Marzwell NI, Uecker DR, Wang Y. A force-controllable macro-micro manipulator and its application to medical robotics [technical report]. Anaheim (CA): Jet Propulsion Laboratory; 1993. Available: http://hdl.handle.net/2014/36461 (accessed 2009 May 7).
9. Tayama T, Olsen DB, Ohashi T, et al. The DeBakey ventricular assist device: current status in 1997. Artif Organs 1999;23:1113-6.

10. Kaya S, Pultz TJ, Mogo CM, et al. The use of radar remote sensing for identifying environmental factors associated with malaria risk in coastal Kenya. Proceeding from the International Geoscience and Remote Sensing Symposium (IGARSS'02); 2002 Jun 24-28; Toronto (ON).

11. Sutherland GR, McBeth PB, Louw DF. NeuroArm: An MR Compatible Robot for Microsurgery. Proceedings of the 17th International Congress and Exhibition; 2003 Jun 25-28; London (UK). Computer Assisted Radiology and Surgery (CARS 2003;1256:504-8.

Correspondence to: Dr. David Williams,Department of Surgery, McMaster University, clo St Joseph's Healthcare Hamilton, 50 Charlton St. E, Hamilton ON L8N 4A6; fax 905 521-6197; willd@mcmaster.ca

While preparing this article, astronaut Dr. Robert Thirsk was getting ready for a 6-month stint on the International Space Station, the first long-duration mission by a Canadian. In addition to being the medical officer for the 6-member international crew, Dr. Thirsk is working as a robotics specialist, operating Canadarm2, and performing experiments on behalf of Canadian and international scientists. He left for the station May 27 aboard a Russian Soyuz rocket from Baïkonur, Kazakhstan.

\section{Articles to date in this series}

- Thirsk R, Kuipers A, Mukai C, et al. The space-flight environment: the International Space Station and beyond. CMAJ 2009;180:1216-20.

- Williams R, Kuipers A, Mukai C, et al. Acclimation in spaceflight: effects on human physiology CMAJ 2009;180: 1317-23.

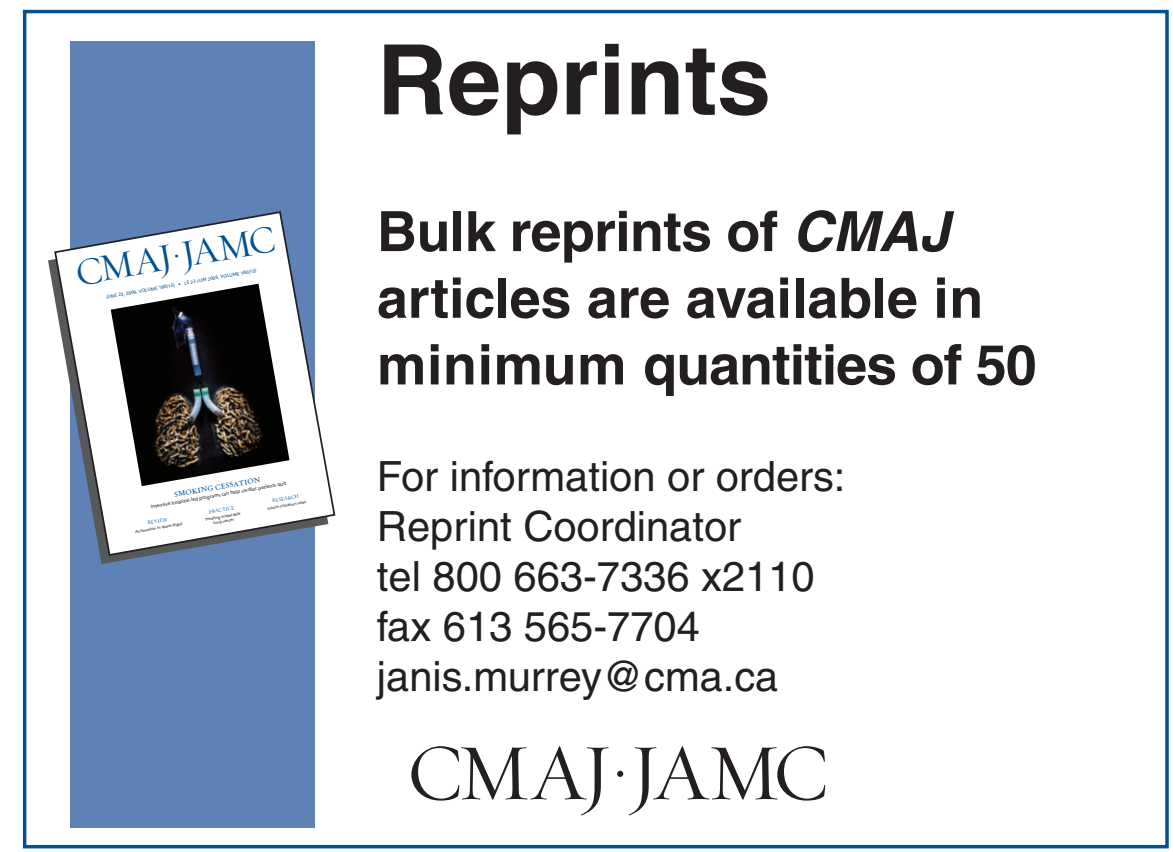

